\title{
Serum Total Bilirubin and Long-Term Prognosis of Patients With New-Onset Non-ST Elevation Myocardial Infarction: A Cohort Study
}

\section{Yi Yang}

the Xinjiang Medical University Affiliated Hospital of Traditional Chinese Medicine Jun Wang

The People's Hospital of Xuancheng City

\section{A Ji Gu Li Wai Si Ding}

the seventh Affiliated Hospital of Xinjiang Medical University

\section{Yanan Xu}

The People's Hospital of Xuancheng City

\section{Haibing Jiang}

the Xinjiang Medical University Affiliated Hospital of Traditional Chinese Medicine

\section{Kezhong Ma}

Xiangyang Central Hospital, Affiliated Hospital of Hubei University of Arts and Science

\section{Tongjian Zhu ( $\nabla$ whuzhutongjian@126.com )}

Xiangyang Central Hospital, Affiliated Hospital of Hubei University of Arts and Science

\section{Research Article}

Keywords: cohort study, major adverse cardiac and cerebrovascular events, non-ST elevation myocardial infarction, SYNTAX scores, total bilirubin

Posted Date: November 12th, 2021

DOI: https://doi.org/10.21203/rs.3.rs-1062415/v1

License: (a) (1) This work is licensed under a Creative Commons Attribution 4.0 International License. Read Full License

Version of Record: A version of this preprint was published at BMC Cardiovascular Disorders on April 12th, 2022. See the published version at https://doi.org/10.1186/s12872-022-02607-8. 
5 Authors: Yi Yang b,1, PhD; Jun Wang c, ${ }^{\mathrm{c}}$, MD; A Ji Gu Li Wai Si Ding e ${ }^{\mathrm{e}, 1}$, MD; Yanan 6

$\mathrm{Xu}^{\mathrm{d}}$, MD; Haibing Jiang a; Kezhong $\mathrm{Ma}^{\mathrm{a}, *}, \mathrm{MD}$; Tongjian $\mathrm{Zhu}{ }^{\mathrm{a}, *}, \mathrm{MD}, \mathrm{PhD}$

\section{Affiliations:}

a Department of Cardiology, Xiangyang Central Hospital, Affiliated Hospital of Hubei University of Arts and Science, Xiangyang, Hubei, 441021, China;

${ }^{\mathrm{b}}$ Department of Cardiology fourth ward, the Xinjiang Medical University Affiliated

Hospital of Traditional Chinese Medicine ; Urumqi 830011,China;

${ }^{\mathrm{c}}$ Department of Cardiology, The People's Hospital of Xuancheng City ; Anhui 242000, China;

${ }^{\mathrm{d}}$ Respiratory medicine, the People's Hospital of Xuancheng City; Anhui 242000,

5 China;

6 e Department of Coronary Heart Disease, the seventh Affiliated Hospital of Xinjiang

17 Medical University, Urumqi 830011, China

\section{* Co-corresponding Author :}

Tongjian Zhu, MD, PhD 
Department of Cardiology, Xiangyang Central Hospital, Affiliated Hospital of Hubei

University of Arts and Science, Xiangyang, Hubei, 441021, China;

Email:whuzhutongjian@126.com

Kezhong Ma, MD

Department of Cardiology, Xiangyang Central Hospital, Affiliated Hospital of Hubei

University of Arts and Science, Xiangyang, Hubei, 441021, China;

E-mail: makezhong2020@163.com

${ }^{1}$ These authors contributed to the work equally and should be regarded as co-first authors. 


\section{Abstract}

Background: The potential prognostic role of total bilirubin (TBIL) in patients with new-onset non-ST elevation myocardial infarction (NSTEMI) is not fully understood. This study aims to evaluate the potential predictive value of TBIL for long-term prognosis in patients with new-onset NSTEMI.

Methods: Patients with new-onset NSTEMI that underwent emergency coronary angiography in our department from June 2015 to March 2020 were included. Baseline TBIL was measured at admission. SYNTAX scores were used to indicate the severity of coronary lesions. The association between TBIL and SYNTAX scores was analyzed using multivariate logistic regression. The patients were followed for the incidence of major adverse cardiac and cerebrovascular events (MACCEs). The association between TBIL and MACCEs was analyzed using Kaplan-Meier survival methods.

Results: In total 327 patients were included in this study. Patients were divided according to tertiles of TBIL (first tertile $<10.23 \mu \mathrm{mol} / \mathrm{L}, \mathrm{n}=109$; second tertile 10.23 - $14.30 \mu \mathrm{mol} / \mathrm{L}, \mathrm{n}=109$; and third tertile $\geq 14.30 \mu \mathrm{mol} / \mathrm{L}, \mathrm{n}=109)$ TBIL was independently associated with the severity of coronary lesions in patients with NSTEMI, with an adjusted odds ratio (OR) and 95\% confidence interval (CI) for the third tertile and the second tertile compared with the first tertile of TBIL of 2.259 (1.197-4.263) and $2.167(1.157-4.059)$, respectively (both $p<0.05)$. After a mean follow-up of 30.33 months, MACCE had occurred in 57 patients. TBIL was 
independently associated with the increased risk of MACCEs, with an adjusted hazard ratio (HR) and 95\% CI for the third tertile and the second tertile compared with the first tertile of TBIL of $2.737(1.161-6.450)$ and 3.272 (1.408-7.607), respectively (both $p<0.05$ ).

Conclusions: Higher myocardial infarction admission TBIL might independently predict poor prognosis in patients with NSTEMI.

Keywords: cohort study; major adverse cardiac and cerebrovascular events; non-ST elevation myocardial infarction; SYNTAX scores; total bilirubin.

60 


\section{Introduction}

The pathological features of acute coronary syndrome (ACS) involve inflammation and oxidative stress that have been associated with conventional risk factors for coronary artery disease (CAD), such as diabetes mellitus, smoking, and hypertension

${ }^{[1-3]}$. However, the evidence suggests that some individuals without the previous risk factors could develop ACS, which suggests that there are potential unknown risk factors for CAD in these patients ${ }^{[4-6]}$. Clinically, non-ST elevation myocardial infarction (NSTEMI) is a subtype of non-ST elevation ACS (NSTE-ACS), which usually is associated with a more severe clinical status and worse outcomes than patients with unstable angina (UA), the other subtype of NSTEACS ${ }^{[7]}$. Therefore, identification of the novel risk factors that might predict the prognosis in patients with NSTEMI is of important clinical significance in current cardiovascular practice.

Previous studies have confirmed that bilirubin, which is a product of heme metabolism, could potentially exert endogenous anti-oxidative and anti-inflammatory efficacies at the physiological level ${ }^{[8]}$. Under pathological conditions, bilirubin could modulate the progression of atherosclerosis by the inhibition of the oxidative modification of low-density lipoprotein and proliferation of smooth muscle cells (SMC) ${ }^{[8]}$. However, elevated bilirubin post-myocardial infarction might reflect increased heme breakdown that includes increased red cell mass, heme oxygenase 1 enzyme (HO-1) expression, myoglobin breakdown, and decreased hepatic bilirubin glucuronidation, or both 
caused by reduced hepatic blood flow following myocardial infarction ${ }^{[9]}$. Therefore, previous clinical studies have suggested that higher serum levels of total, direct, and indirect bilirubin might be associated with an increased risk of the combined outcomes of major adverse cardiac and cerebrovascular events (MACCEs) in patients with ACS, which include all-cause death, myocardial infarction, and stroke ${ }^{[10-11]}$. However, some of the previous studies have indicated that total bilirubin (TBIL) might confer better prognostic efficacy than direct or indirect bilirubin in ACS patients ${ }^{[12-13]}$, other studies that evaluated the predictive role of serum TBIL in ACS patients based on the subtype of ACS showed inconsistent results ${ }^{[11,13-15]}$. Some of the studies did not support that serum TBIL was associated with an increased risk of MACCEs in ACS patients ${ }^{[14-15] .}$ In addition, the sample sizes of previous studies were limited, and patients with previously diagnosed CAD were included, which might affect the results of the studies. Because of the important role of inflammation in the pathogenesis of NSTEMI, as well as the potential role of bilirubin as an endogenous anti-inflammatory factor, this study aims to systematically evaluate the potential associations between serum TBIL with severity and prognosis in patients with new-onset NSTEMI.

\section{Methods}

\section{Patients and study design}

Patients with new-onset NSTEMI and without previously known CAD that underwent urgent coronary angiography in the Xinjiang Medical University Affiliated Hospital of 
Traditional Chinese Medicine from June 2015 to March 2020 were included in this study. The diagnosis of NSTEMI was based on the criteria in previous guidelines ${ }^{[17]}$. Patients with any of the following clinical conditions were excluded: (1) hepatic or renal dysfunction that might affect serum TBIL; (2) diagnosis of ST-segment elevation myocardial infarction (STEMI), unstable angina pectoris, or with previous revascularization therapy, which included percutaneous coronary intervention (PCI) and coronary artery bypass grafting (CABG); (3) new-onset NSTEMI with a previous diagnosis of $\mathrm{CAD}$; (4) patients that had pacemaker-implantation, malignant tumors, or severe infection; (5) patients with previously diagnosed systemic inflammatory disease, a history of alcohol consumption, hemolysis, blood transfusion, viral infections of the liver, or with poor compliance to treatment; and (6) patients who were at risk of hepatotoxicity induced by medications, such as the use of statins or amidarone. The flow chart of participant enrollment is shown in Fig. 1.

\section{Blood sampling}

Peripheral venous blood samples were drawn immediately before urgent coronary angiography for each of the patients and sent to the Department of Clinical Laboratory of the Xinjiang Medical University Affiliated Hospital of Traditional Chinese Medicine for further analysis. Parameters of blood cell count, biochemical parameters for lipids and glucose metabolism, hepatic and renal function, serum uric acid, serum creatine phosphokinase-MB (CK-MB), and troponin T were measured. 
After admission, all patients underwent emergency coronary angiography using a standard protocol that was carried out by experienced cardiologists. The SYNTAX score was used as the indicator for the severity of the coronary lesions, which was calculated by two experienced cardiologists independently according to the online tool of the score. If disagreement occurred, they were resolved by consensus with the third investigator. If indications for PCI were detected, the modality of PCI was determined by a group of experienced attending physicians based on coronary anatomy and the clinical status of the individual patients. After PCI, patients continued with optimized medical treatments and were followed-up at clinics regularly after discharge.

\section{Follow-up}

Patients were discharged and followed-up by telephone interview or clinic visits. All events were carefully monitored by an independent panel of clinical physicians. The primary outcome of this study was the incidence of a combined outcome for MACCEs, which included cardiac mortality, myocardial infarction, stent thrombosis, stroke and revascularization. The secondary outcome of this study was all-cause mortality.

\section{Statistical analysis}

Patients were grouped based on the tertiles of the serum TBIL (first tertile $<10.23$ $\mu \mathrm{mol} / \mathrm{L}$, second tertile $10.23-14.30 \mu \mathrm{mol} / \mathrm{L}$, and third tertile $\geq 14.30 \mu \mathrm{mol} / \mathrm{L}$, with 109 patients in each tertile) or tertiles of the SYNTAX score at baseline. Continuous 
variables were summarized as mean and standard deviation if normally distributed; otherwise, medians and interquartile ranges (IQRs) were used. Categorical variables were expressed as percentages. Comparisons with means between multiple groups were performed using ANOVA, and for the nonnormally distributed variables, Mann-Whitney U test or Kruskal-Wallis variance analysis was applied. For the categorical variables, a Chi-squared ( $\chi 2)$ test was employed. Multiple logistic regression analysis was performed to identify the independent factors that were associated with the severity of coronary lesions, as evidenced by the SYNTAX score. The potential predictive efficacy of serum TBIL at baseline for prognosis in NSTEMI patients was analyzed using the Kaplan-Meier survival method. Univariate analysis was performed first, and then the significant variables were included in the multivariate Cox regression analysis. A $p$-value $<0.05$ indicated a statistically significant difference. SPSS 23 was used for the statistical analysis.

\section{Results}

\section{Characteristics of the included patients}

In total, 327 patients with new-onset NSTEMI were retrospectively included in this study. The baseline characteristics for all the patients included in this study based on the tertiles of TBIL (first tertile $<10.23 \mu \mathrm{mol} / \mathrm{L}, \mathrm{n}=109$; second tertile 10.23-14.30 $\mu \mathrm{mol} / \mathrm{L}, \mathrm{n}=109$; and third tertile $\geq 14.30 \mu \mathrm{mol} / \mathrm{L}, \mathrm{n}=109)$ are presented in Table 1. The results showed that patients with higher TBIL levels were probable to be male and 
smokers, with higher apolipoprotein A1, (Apo-AI), increased high-density

162 lipoprotein cholesterol, and a higher SYNTAX score (all $p<0.05$ ). The baseline 163 characteristics for all the patients in this study were based on tertiles of the SYNTAX 164 score as given in Table 2. Age, the prevalence of diabetes mellitus, smoking status, 165 left ventricle ejection fraction (LVEF), TBIL, and TBIL tertiles group were 166 significantly different between the patients based on the tertiles of SYNTAX score (all $167 p<0.05)$.

\section{Potential association between TBIL and severity of coronary lesions}

169 The results of multivariate logistic analyses showed that a higher TBIL was 170 independently associated with the severity of coronary lesions based on the SYNTAX 171 score, with an adjusted odds ratio (OR) and 95\% confidence interval (CI) for the third 172 tertile and the second tertile compared with the first tertile of TBIL of 2.259 (1.1974.263) and $2.167(1.157-4.059)$, respectively as given in Table 3 (both $p<0.05)$ In

174 addition, other factors that include diabetes (OR: 1.954, $p=0.016$ ), smoker (OR: $1751.829, p=0.023$ ), and LVEF (OR: 0.954, $\mathrm{p}=0.032$; Table 3) were associated with the 176 severity of coronary lesions in patients with new-onset NSTEMI. Incidence of MACCEs and all-cause mortality according to the TBIL

178 The incidences of primary and secondary clinical outcomes during a mean follow-up 179 of 30.33 months for the patients in this study with new-onset NSTEMI, based on the 180 tertiles of TBIL at baseline are given in Table 4. During follow-up, 57 patients 
experienced MACCEs. The results showed that the incidence of MACCEs increased in patients based on the tertiles of serum levels of TBIL $(p=0.001)$. However, the incidence of all-cause mortality was not statistically different between patients with new-onset NSTEMI, based on the tertiles of TBIL at baseline $(p=0.177)$.

\section{Predictors of clinical outcomes}

Kaplan-Meier analysis demonstrated that the incidence of MACCEs was significantly different among patients with new-onset NSTEMI based on the tertiles of TBIL $(\chi 2=15.243, p<0.001)$ as shown in Fig. 2 and the incidence of all-cause mortality was not significantly different among patients based on the tertiles of TBIL ( $\chi^{2}=4.430$, $\mathrm{p}=0.109$ ) as shown in Fig. 3. The Results of univariate Cox regression analysis indicated that gender (female), hypertension, diabetes, increased troponin $\mathrm{T}$, unprotected left main trunk coronary artery lesions, higher TBIL tertile, and higher SYNTAX score tertile were potential predictors of MACCEs, as given in Table 5 ( $p$-values all $<0.05)$. Subsequent multivariate analysis showed that TBIL was independently associated with an increased risk of MACCEs, with adjusted hazard ratio (HR) and 95\% CI for the third tertile and the second tertile compared with the first tertile of TBIL of 2.737 (1.161 -6.450) and 3.272 (1.408-7.607), respectively (both $\mathrm{p}<0.05$ ). Other independent risk factors for the increased incidence of MACCEs in patients with new-onset NSTEMI included diabetes (HR: 1.800, 95\% CI: 1.041-3.113, $\mathrm{p}=0.035$ ), UPLMCA (HR: 2.042, 95\% CI: $1.063-3.923, p=0.032$ ), 
increased troponin T (HR: 1.172, 95\% CI: 1.007-1.365, p =0.040), and increased

SYNTAX scores (third tertile versus first tertile, HR: 3.165 , 95\% CI: $1.280-7.827$, $\mathrm{p}=0.013$ ); and second tertile versus first tertile (HR: 2.767, 95\% CI: 1.097-6.980, $\mathrm{p}=$ 0.031 ) as given in Table $\mathbf{5}$.

\section{Discussion}

In this retrospective cohort study that included patients with new-onset NSTEMI, a

higher TBIL at baseline was independently associated with the severity of coronary

lesions as shown by the higher SYNTAX score. In addition, with a mean follow-up of 30.33 months, higher serum TBIL at baseline was an independent predictor for an

210 increased incidence of MACCEs in patients with new-onset NSTEMI. Because of the

211 convenience and cost-effectiveness of measuring myocardial infarction admission

212 TBIL in clinical practice, these results suggested that serum TBIL might be an

213 inexpensive predictor for poor prognosis in patients with new-onset NSTEMI.

214 The risk stratification for patients with new-onset NSTEMI needs to be improved, in

215 particular, for the identification of potential prognostic factors for these patients ${ }^{[7]}$.

216 Although previous studies have suggested a potential role of TBIL as a prognostic

217 factor in $\mathrm{CAD}$, the results of these studies might be different based on the subtype of

218 CAD. A previous study that included 7,685 healthy individuals with a mean follow-up

219 of 11.5 years showed that higher TBIL might be a risk factor for the increased

220 incidence of ischemic heart disease ${ }^{[18]}$. A retrospective study that included 3,013 
patients with angiographically obstructive CAD suggested a positive and independent correlation between baseline levels of TBIL and short-term mortality of acute myocardial infarction patients, and the negative correlation between baseline levels of

TBIL and long-term mortality in stable CAD or UA pectoris patients was confirmed in a cohort study with a follow-up of 1 year ${ }^{[19]}$. In addition, high serum TBIL levels have been independently and significantly correlated with the burden of coronary atherosclerosis in patients with STEMI, and no significant association between high serum TBIL levels and poor long-term prognosis was found in these studies ${ }^{[15,20]}$.

In this retrospective cohort study, a significant association was found between myocardial infarction admission TBIL and the severity of coronary lesions. In addition, higher myocardial infarction admission TBIL was independently associated with a higher risk of MACCEs in new-onset NSTEMI patients with a mean follow-up of 30.33 months. However, TBIL might be a potential protective factor for coronary lesions based on the potential endogenous anti-oxidative and anti-inflammatory characteristics of bilirubin. These findings agreed with a previous hypothesis that myocardial infarction admission TBIL was elevated shortly after myocardial infarction because of the acute response to impaired liver function ${ }^{[21]}$. In addition, previous studies demonstrated that the TBIL changed dramatically as regulated by HO-1 and the maximal levels of bilirubin were usually observed during an acute myocardial infarction event ${ }^{[22-23]}$. Moreover, STEMI patients with high bilirubin levels were shown to have a higher incidence of adverse outcomes and mortality during 
hospitalization ${ }^{[24]}$, which suggested a role for increased TBIL as a predictor for poor prognosis in STEMI patients. This study, by strictly excluding patients with a previous diagnosis of CAD and other concurrent comorbidities that might affect the TBIL level, showed that higher TBIL at baseline was independently associated with a higher risk of MACCEs in new-onset NSTEMI patients. These findings support the incorporation of baseline TBIL levels for risk stratification of patients with new-onset NSTEMI.

The pathophysiological mechanisms that underly the association between bilirubin and poor prognosis in patients with new-onset NSTEMI need to be determined. Based on this study, it could be hypothesized that acute myocardial ischemia might induce an immediate increase in the levels of various inflammatory cytokines and reduced hepatic blood flow, which might exceed the protective antioxidant effect of bilirubin in vivo. In addition, it could be inferred that there was a compensatory increase of TBIL by dramatically up-regulated HO-1 activity under stress to exert anti-inflammatory and anti-oxidative effects in new-onset NSTEMI patients. This is consistent with previous findings that patients with increased serum bilirubin levels had increased cardiac troponin I release that was correlated with myocardial infarction size and the severity of coronary atherosclerotic burden ${ }^{[25]}$. Therefore, high TBIL levels might have a protective anti-oxidative effect on the cardiovascular system in stable CAD and healthy population. In addition, it has been suggested that long-term therapy with statins or aspirin might be associated with increased TBIL levels ${ }^{[26-27]}$. However, this appears not to be the main influencing factors in this study, because only new-onset 
NSTEMI patients without a previous diagnosis of CAD were included.

264

265

266

267

\section{Study limitations}

Several limitations of this study are noted. First, this was a retrospective observational study with limited sample size, and the findings should be validated in large-scale prospective cohort studies. In addition, the serum TBIL was only measured once at admission, and whether dynamic changes in serum TBIL during hospitalization had a more significant impact on the prognosis of these patients is unknown. In addition, this was an observational study, and a causative association between increased serum TBIL and poor prognosis in these patients could not be derived based on the findings. Finally, the optimal cut-off value for the prognostic efficacy of TBIL is unknown, which deserves further investigation.

\section{Conclusions}

The results of this study suggest that myocardial infarction admission TBIL might be an inexpensive predictor of poor prognosis in patients with new-onset NSTEMI. Because of the convenience and cost-effectiveness of measuring serum TBIL, the findings support the incorporation of the measurement of serum TBIL when risk stratification for patients with new-onset NSTEMI is performed. 


\section{Abbreviations}

$\mathrm{ACEI}=$ angiotensin-converting enzyme inhibitor; Apo-AI = apolipoprotein A1; Apo-B

284 = apolipoprotein $\mathrm{B} ; \mathrm{ARB}=$ angiotensin II receptor blocker; $\mathrm{BMI}=$ body mass index;

$285 \mathrm{BUN}=$ blood urea nitrogen; $\mathrm{CCB}=$ calcium channel blocker; $\mathrm{Cr}=$ creatinine; $\mathrm{CK}-\mathrm{MB}$

$286=$ creatine kinase-MB; $\mathrm{DBP}=$ diastolic blood pressure; HDL-C $=$ high-density

287 lipoprotein cholesterol; LAD = left anterior descending artery; LCX = left circumflex

288 artery; LDL-C = low-density lipoprotein cholesterol; LVEDD = left ventricular

289 end-diastolic dimension; $\mathrm{LVEF}=$ left ventricle ejection fraction; $\mathrm{PCI}=$ percutaneous

290 coronary intervention; RCA = right coronary artery; SBP = systolic blood pressure; TC

$291=$ total cholesterol; $\mathrm{TG}=$ triglyceride; and UPLMT $=$ unprotected left main trunk.

\section{Declarations}

293 The authors declare that they have no conflict of interests.

\section{Ethical approval}

295 Because this was a retrospective observational study, the ethics committee of 296 the Xinjiang Medical University Affiliated Hospital of Traditional Chinese Medicine 297 granted an exemption from ethics approval.

\section{Informed consent}

299 Because this was a retrospective observational study, the ethics committee of 300 the Xinjiang Medical University Affiliated Hospital of Traditional Chinese Medicine 301 waived the requirement for informed consent from eligible patients.

\section{Consent for publication:}


Not applicable.

Availability of data and material

The datasets used and/or analyzed during this study are available from the corresponding author on reasonable request. Requests to access these datasets should be directed to Tongjian Zhu, whuzhutongjian@126.com.

\section{Competing Interest:}

309 The authors declare that they have no conflict of interest.

\section{Funding:}

311 None of.

312 Authors' contributions:

313 Conceived and designed the study: TZ and KM. Data collection and analyzed the

314 data:YY.Quality control the study and revision: JW and YX. Wrote the paper: YY and

315 AD. YY, JW and AD contributed to the work equally and should be regarded as

316 co-first authors. The manuscript was approved by all above authors.

\section{Acknowledgments}

318 Thanks to everyone for supporting our final study. 


\section{References}

327

328

329

330

331

332

333

334

335

336

337

[1] Ridker PM, Everett BM, Thuren T, et al. Antiinflammatory Therapy with Canakinumab for Atherosclerotic Disease. 2017; N Engl J Med. 377: 1119-31.

[2] Jun W,Lu J,Xing L, et al. New Insights into the Association between Fibrinogen and Coronary Atherosclerotic Plaque Vulnerability: An Intravascular Optical Coherence Tomography Study.[J] .Cardiovasc Ther, 2019, 2019: 8563717.

[3] Khot UN, Khot MB, Bajzer CT, et al. Prevalence of conventional risk factors in patients with coronary heart disease. JAMA. 2003;290:898-904

[4] Greenland P, Knoll MD, Stamler J, et al.Major risk factors as antecedents of fatal and nonfatal coronary heart disease events. JAMA.2003; 290: 891-897.

[5] Yi Y,Yanan X ,Jun W, et al. Predictive efficacy of neutrophil-to-lymphocyte ratio for long-term prognosis in new onset acute coronary syndrome: a retrospective cohort study.[J] .BMC Cardiovasc Disord, 2020, 20: 500.

[6] Jun W, Xing L,Jun P, et al. Mean platelet volume and coronary plaque vulnerability: an optical coherence tomography study in patients with non-ST-elevation acute coronary syndrome.[J] .BMC Cardiovasc Disord, 2019, 19: 128. 
343

344

345

346

347

348

349

350

351

352

353

354

355

356

357

358

359

360

361

362

[7] Tziakas D, Chalikias G, Al-Lamee R, et al. Total coronary occlusion in non ST

elevation myocardial infarction: Time to change our practice?[J] .Int J Cardiol, 2021, 329: 1-8.

[8] Drummond Heather A, Mitchell Zachary L, Abraham Nader G, et al. Targeting Heme Oxygenase-1 in Cardiovascular and Kidney Disease.[J] .Antioxidants (Basel), 2019 18;8(6):181.

[9] Kunutsor S K, Bakker S J, Gansevoort R T, et al. Circulating Total Bilirubin and Risk of Incident Cardiovascular Disease in the General Population.[J]. Arterioscler Thromb Vasc Biol, 2015, 35(3):716-724.

[10] Ochoa E L,Wennberg R P, An Y et al. Interactions of bilirubin with isolated presynaptic nerve terminals: functional effects on the uptake and release of neurotransmitters.[J] .Cell Mol Neurobiol, 1993, 13: 69-86.

[11] Kunutsor S K , Kieneker L M, Burgess S, et al. Circulating Total Bilirubin and Future Risk of Hypertension in the General Population: The Prevention of Renal and Vascular End-Stage Disease (PREVEND) Prospective Study and a Mendelian Randomization Approach[J]. Journal of the American Heart Association, 2017, 6(11):e006503.

[12] Huang F Y , Peng Y, Huang B T , et al. The correlation between serum total bilirubin and outcomes in patients with different subtypes of coronary artery disease[J]. Clin Chim Acta, 2017, 465: 101-105. 
[13] Allen Larry A, Felker G Michael, Pocock Stuart et al. Liver function

364 abnormalities and outcome in patients with chronic heart failure: data from the

365 Candesartan in Heart Failure: Assessment of Reduction in Mortality and Morbidity

366 (CHARM) program.[J] .Eur J Heart Fail, 2009, 11: 170-7.

367 [14] Chenbo X,Mengya D, Yangyang D,et al. Relation of Direct, Indirect, and Total 368 bilirubin to Adverse Long-term Outcomes Among Patients With Acute Coronary 369 Syndrome.[J] .Am J Cardiol, 2019, 123: 1244-1248.

[15] Xiaoxiao Z, Ying W, Chen L ,et al. Prognostic Value of Total Bilirubin in Patients

With ST-Segment Elevation Acute Myocardial Infarction Undergoing Primary

372 Coronary Intervention.[J] .Front Cardiovasc Med, 2020, 7: 615254.

373 [16] Kaya Mehmet G,Sahin O,Akpek M et al. Relation between serum total bilirubin 374 levels and severity of coronary artery disease in patients with non-ST-segment 375 elevation myocardial infarction.[J] .Angiology, 2014, 65: 245-9.

376 [17] Goldfine Allison B,Shoelson Steven E. Therapeutic approaches targeting 377 inflammation for diabetes and associated cardiovascular risk.[J] .J. Clin. Invest., 2017, $378 \quad 127: 83-93$.

379 [18] Mendis S,Thygesen K,Koulasmaa K,et a1. Wodd Health Organization definition 380 of myocardial infarction: 2008-09 revision [J]. Int J Epidemiol, 2011, 40(1): $381139 \cdot 146$. DOI: $10.1093 /$ ije / dyq 165. 
382

383

384

385

386

387

[19] Breimer L H,Wannamethee G,Ebrahim S et al. Serum bilirubin and risk of ischemic heart disease in middle-aged British men.[J] .Clin Chem, 1995, 41: 1504-8.

[20] Fang-Yang H,Yong P,Bao-Tao $\mathrm{H}$ et al. The correlation between serum total bilirubin and outcomes in patients with different subtypes of coronary artery disease.[J] .Clin Chim Acta, 2017, 465: 101-105.

[21] Sahin O,Akpek M,Elcik D,et al. Bilirubin levels and the burden of coronary atherosclerosis in patients with STEMI.[J] .Angiology, 2013, 64: 200-4.

[22] Bulmer Andrew C, Bakrania Bhavisha, Du Toit Eugene F et al. Bilirubin acts as a multipotent guardian of cardiovascular integrity: more than just a radical idea.[J] .Am J Physiol Heart Circ Physiol, 2018, 315: H429-H447.

[23] Okuhara K, Kisaka T, Ozono R, et al. Change in bilirubin level following acute myocardial infarction is an index for heme oxygenase activation.[J] .South Med J, 2010, 103: 876-81.

[24] Lakkisto P, Palojoki E, Bäcklund T, et al. Expression of heme oxygenase-1 in response to myocardial infarction in rats.[J] .J Mol Cell Cardiol, 2002, 34: 1357-65.

[25] Gul M, Uyarel H, Ergelen M, et al. Prognostic value of total bilirubin in patients with ST-segment elevation acute myocardial infarction undergoing primary coronary intervention.[J] .Am J Cardiol, 2013, 111: 166-71. 
400 [26] Ozturk M, Askkk L, Ipek E et al. The Role of Serum Bilirubin Levels in 401 Predicting Troponin Positivity in Non-ST-Segment Elevation Acute Coronary 402 Syndrome.[J] .Angiology, 2017, 68: 414-418.

403 [27] de Sauvage Nolting Pernette R W, Kusters D Meeike, Hutten Barbara A, et al. 404 Serum bilirubin levels in familial hypercholesterolemia: a new risk marker for 405 cardiovascular disease?[J] .J Lipid Res, 2011, 52: 1755-9.

406 [28] Canpolat U,Cagli K,Basar Fatma N, et al. The prognostic role of serum total 407 bilirubin in non-ST segment elevation myocardial infarction: what about on-admission 408 cardiovascular medications?[J] .Angiology, 2014, 65: 250.

409 
Figure 1. Flowchart of patient enrollment.

Figure 2. Cumulative incidence of MACCE in patients with NSTEMI according to TBIL tertiles.

Figure 3. Cumulative incidence of all-cause mortality in patients with NSTEMI according to TBIL tertiles. 
Figures

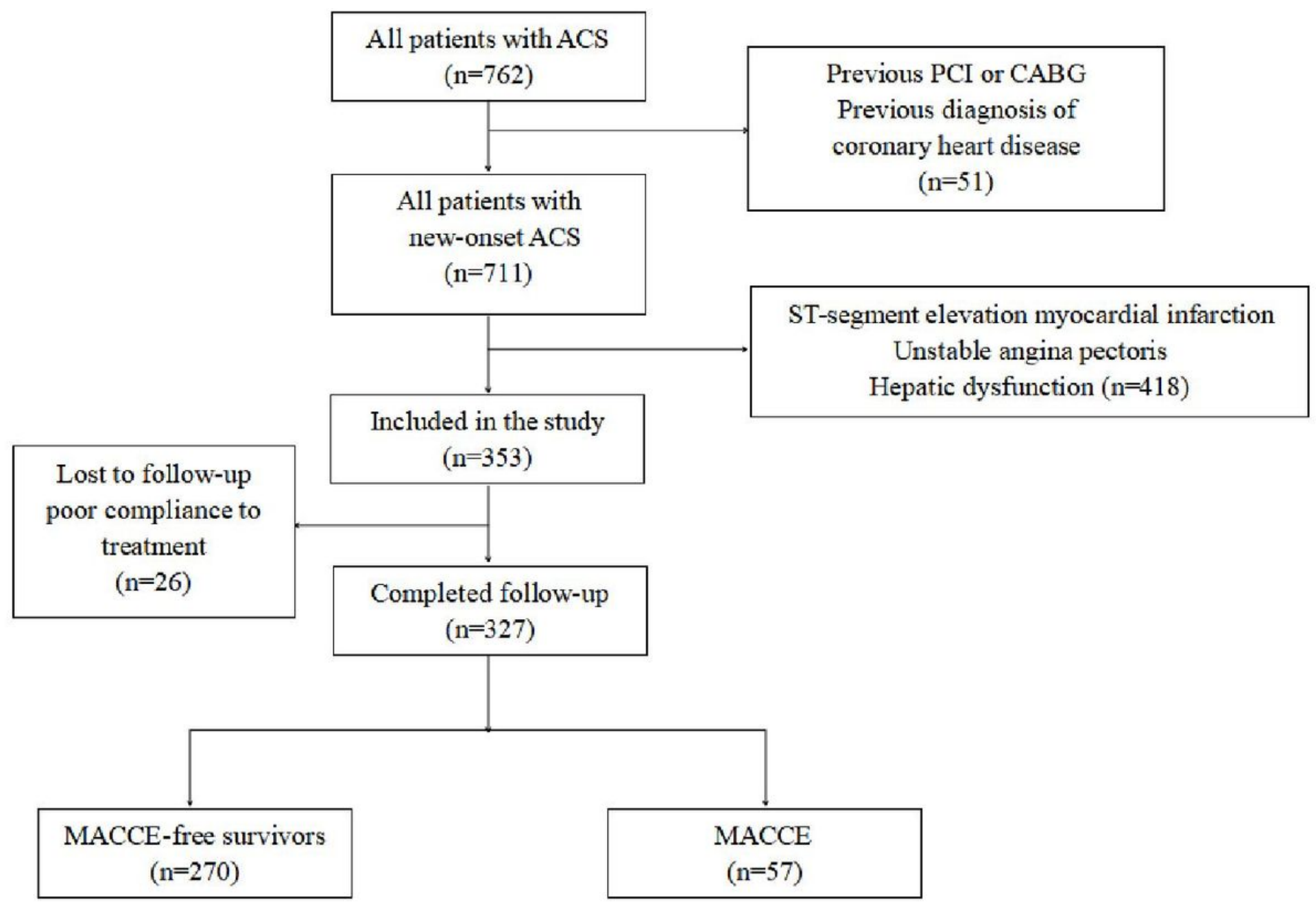

Figure 1

Flowchart of patient enrollment. 


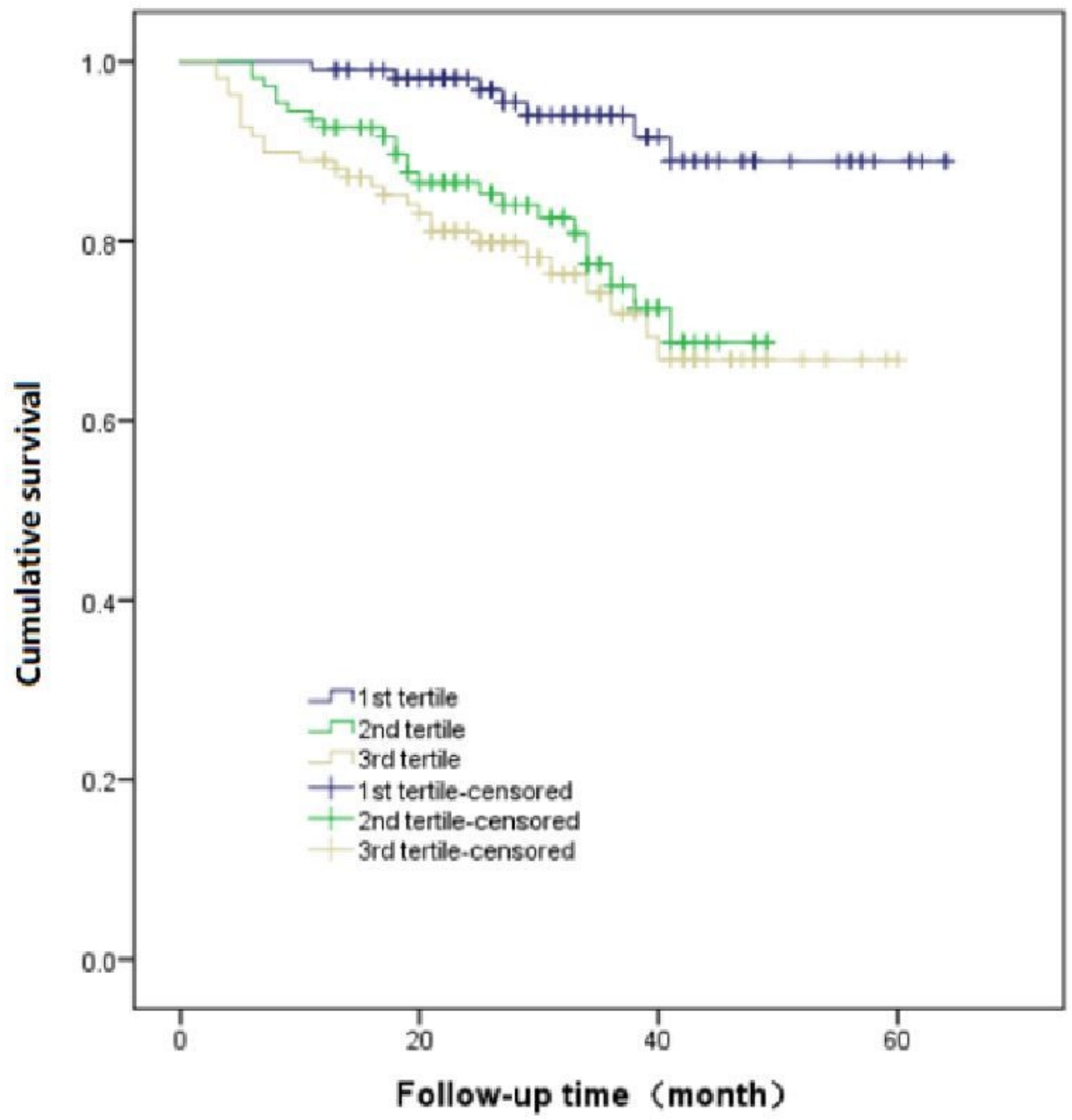

Figure 2

Cumulative incidence of MACCE in patients with NSTEMI according to TBIL tertiles. 


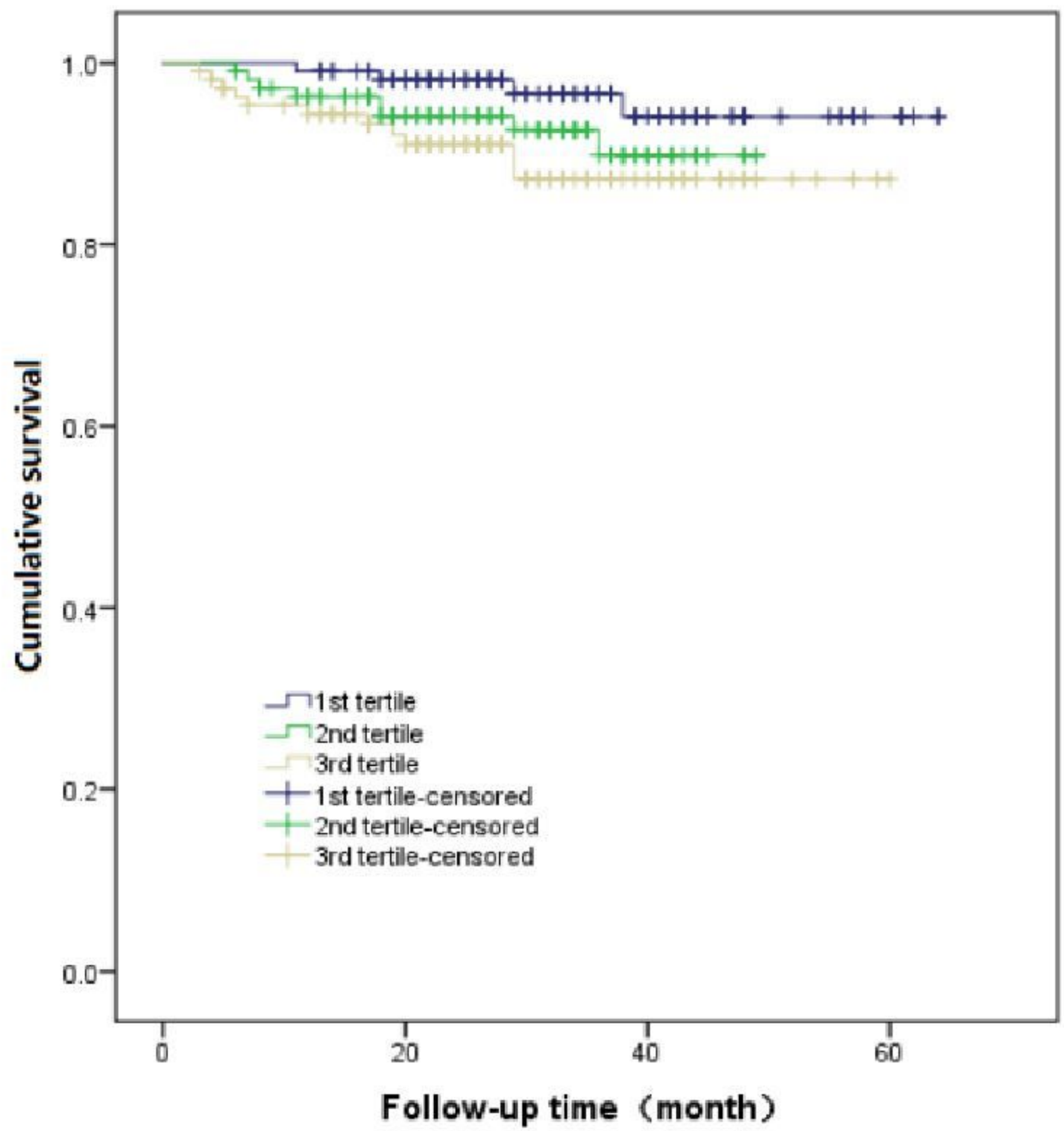

Figure 3

Cumulative incidence of all-cause mortality in patients with NSTEMI according to TBIL tertiles. 